\title{
Random matrix theory for CPA: Generalization of Wegner's $n$-orbital model
}

\author{
Peter $\mathrm{Neu}^{1}$ and Roland Speicher ${ }^{2} \dagger$ \\ ${ }^{1}$ Institut für Theoretische Physik, Universität Heidelberg, Philosophenweg 19, 69120 Heidelberg, Germany \\ 2 Institut für Angewandte Mathematik, Universität Heidelberg, Im Neuenheimer Feld 294, 69120 Heidelberg, Germany
}

(August 26, 1994)

We introduce a generalization of Wegner's $n$-orbital model for the description of randomly disordered systems by replacing his ensemble of Gaussian random matrices by an ensemble of randomly rotated matrices. We calculate the one- and twoparticle Green's functions and the conductivity exactly in the limit $n \rightarrow \infty$. Our solution solves the CPA-equation of the $(n=1)$-Anderson model for arbitrarily distributed disorder. We show how the Lloyd model is included in our model.

PACS numbers: 71.10.+x, 05.60.+w, 71.55.-i

The treatment of physical systems with disorder presents one of the great challenges in statistical physics. Realistic multi-site models, like the Anderson model [1], are in general unsolvable. Exact calculations are only possible in one pathological special case - namely for the Lloyd model [2] with Cauchy-distributed disorder. Otherwise one has to use approximation techniques which reduce the multi-site to single-site models and treat them self-consistently. The most prominent of these methods is the so-called coherent potential approximation (CPA), see, e.g., [3].

Wegner 4 introduced a generalization of the Anderson model by putting $n$ electronic states at each site and describing the disorder by Gaussian random matrices in these electronic states. Whereas for $n=1$ this reduces to the usual (unsolvable) Anderson model, the opposite limit $n \rightarrow \infty$ becomes exactly solvable. Interestingly, the solution of this multi-site model coincides with a special CPA-solution.

In this letter we introduce a generalization of Wegner's model by replacing his ensemble of Gaussian random matrices by a more general ensemble of random matrices and thus allowing arbitrarily distributed disorder. Nevertheless - by using recent results of Voiculescu [5, 6] and Speicher [7.8] on the mathematical concept of "freeness" - we are still able to calculate the Green's functions of this model in the limit $n \rightarrow \infty$ exactly. Again our solution solves the CPA-equation of the $(n=1)$-Anderson model. Hence our model can be regarded as a rigorous mean-field model for CPA for arbitrarily distributed disorder.

Let us first recall Wegner's model. He considers a $d$ dimensional lattice where at each site $r$ there are $n$ electronic levels $|r \alpha\rangle$ numbered by $\alpha=1, \ldots, n$. The interaction is governed by a Hamiltonian of the form

$$
H=H_{0}+H_{1},
$$

where $H_{0}$ is deterministic, translational-invariant, and diagonal in the electronic levels,

$$
H_{0}=\sum_{r, r^{\prime}, \alpha} v_{r-r^{\prime}}|r \alpha\rangle\left\langle r^{\prime} \alpha\right|
$$

and $H_{1}$ describes the site-diagonal disorder

$$
H_{1}=\sum_{r, \alpha, \beta} \frac{1}{\sqrt{n}} f_{r}^{\alpha \beta}|r \alpha\rangle\langle r \beta| .
$$

There, $f_{r}=\left((1 / \sqrt{n}) f_{r}^{\alpha \beta}\right)_{\alpha, \beta=1}^{n}$ are Gaussian random matrices and the entries of $f_{r}$ and of $f_{r^{\prime}}$ are independent for $r \neq r^{\prime}$. (This is the site-diagonal model of Wegner, we will not treat his "local gauge invariant" model here.) By using techniques for calculating moments of Gaussian random matrices [9, 10], Wegner was able to calculate the one- and two-particle Green's function in the limit $n \rightarrow \infty$.

To explain our generalization let us diagonalize the random matrices $f_{r}$ in the form $f_{r}=u_{r} \hat{f}_{r} u_{r}^{*}$. Thus the ensemble of the $f_{r}$ is determined by an ensemble of diagonal matrices $\hat{f}_{r}$ and an ensemble of unitary matrices $u_{r}$. In the limit $n \rightarrow \infty$, Wegner's original formulation is recovered if the diagonal $\hat{f}_{r}$ are taken as deterministic matrices having Wigner's semi-circle [9] as eigenvalue distribution and if the $u_{r}$ are random unitary matrices, given by the canonical invariant or Haar measure on $U(n)$, such that $u_{r}$ and $u_{r^{\prime}}$ are chosen independently from $U(n)$ for $r \neq r^{\prime}$. Since this ensemble is invariant under independent rotations at different sites we may also replace the different $\hat{f}_{r}$ by one single (not necessarily diagonal) $f$, i.e. we have $f_{r}=u_{r} f u_{r}^{*}$ with the ensemble of $u_{r}$ as stated above.

The advantage of this reformulation of Wegner's model is that now a generalization is obvious: We are a priori totally free in the choice for $f$. Thus our model is given by the following Hamiltonian

$$
H=\sum_{r, r^{\prime}, \alpha} v_{r-r^{\prime}}|r \alpha\rangle\left\langle r^{\prime} \alpha\left|+\sum_{r, \alpha, \beta}\left(u_{r} f u_{r}^{*}\right)_{\alpha, \beta}\right| r \alpha\right\rangle\langle r \beta|,
$$

where $f$ is a deterministic hermitian $n \times n$-matrix and $u_{r} \in U(n)$ are random unitary matrices, chosen independently for different sites. This means that we act at each site $r$ with a copy $f_{r}:=u_{r} f u_{r}^{*}$ of the given operator $f$, but that the basis for $f_{r}$ and the basis for $f_{r^{\prime}}$ are rotated randomly against each other for all pairs of different sites $r \neq r^{\prime}$.

The possibility for treating this model in the limit $n \rightarrow$ $\infty$ arises from the important observation of Voiculescu [5] (see also [11]) that there is a connection with his concept of free random variables [13, 6, 12]: Denote by $\langle\ldots\rangle_{\text {ens }}$ the 
average over our ensemble of random unitary matrices and let

$$
\langle\ldots\rangle:=\left\langle\frac{1}{n} \sum_{\alpha=1}^{n}\langle\alpha|\ldots| \alpha\rangle\right\rangle_{\mathrm{ens}}
$$

count the averaged eigenvalue distribution of our $n \times$ $n$-matrices. Then we have for all $m \in \mathbf{N}$ and for all polynomials $p_{1}, \ldots, p_{m}$ with $\left\langle p_{i}(f)\right\rangle=0(i=1, \ldots, m)$ in the limit $n \rightarrow \infty$ that also

$$
\left\langle p_{1}\left(f_{r(1)}\right) p_{2}\left(f_{r(2)}\right) \ldots p_{m}\left(f_{r(m)}\right)\right\rangle=0
$$

for all sequences of indices $r(1), \ldots, r(m)$, where all consecutive indices are different, i.e. where $r(i) \neq r(i+1)$ for all $i=1, \ldots, m-1$. An example of (6) is

$$
\left\langle p_{1}\left(f_{1}\right) p_{2}\left(f_{2}\right) p_{3}\left(f_{1}\right) p_{4}\left(f_{2}\right)\right\rangle=0
$$

whereas for sequences with coinciding neighbouring indices one gets non-vanishing results like

$$
\left\langle p_{1}\left(f_{1}\right) p_{2}\left(f_{2}\right) p_{3}\left(f_{2}\right) p_{4}\left(f_{1}\right)\right\rangle=
$$

$\left\langle p_{1}\left(f_{1}\right) p_{4}\left(f_{1}\right)\right\rangle\left\langle p_{2}\left(f_{2}\right) p_{3}\left(f_{2}\right)\right\rangle=\left\langle p_{1}(f) p_{4}(f)\right\rangle\left\langle p_{2}(f) p_{3}(f)\right\rangle$.

The property $(6)$ - in the mathematical literature recently introduced by Voiculescu [13,6] under the name of "freeness" - allows, as in Wegner's case, to calculate all mixed moments of the matrices $f_{r}$ (like, e.g., in (8) ) and thus to derive exact expressions for the Green's functions of our model in the limit $n \rightarrow \infty$. Whereas usually one gets an infinite hierarchy of equations for averaged quantities, it is exactly the property of freeness (5) which closes our equations. For an effective handling of the calculations one needs the $R$-transform machinery of Voiculescu [14,6] and the concept of non-crossing cumulants of Speicher [7,8]. The concrete calculations will be published elsewhere, here we only want to give the results.

The most important quantity is (the diagonal part of) the one-particle Green's function (1PG)

$$
G(z):=\left\langle\frac{1}{n} \sum_{\alpha=1}^{n}\left\langle r \alpha\left|\frac{1}{z-H}\right| r \alpha\right\rangle\right\rangle_{\mathrm{ens}}
$$

whose spectral function yields the density of states. Note that, due to the translation-invariance of our Hamiltonian, $G(z)$ is independent of $r$ and hence the local and the global density of states coincide. If we denote similarily by $G_{0}$ and $G_{1}$ the $1 \mathrm{PG}$ of the deterministic and the disorder part of $H$, respectively, i.e.

$$
\begin{aligned}
G_{0}(z) & =\left\langle r\left|\frac{1}{z-H_{0}}\right| r\right\rangle \\
G_{1}(z) & =\left\langle\frac{1}{n} \sum_{\alpha=1}^{n}\left\langle\alpha\left|\frac{1}{z-f_{r}}\right| \alpha\right\rangle\right\rangle_{\mathrm{ens}} \\
& =\frac{1}{n} \sum_{\alpha=1}^{n}\left\langle\alpha\left|\frac{1}{z-f}\right| \alpha\right\rangle,
\end{aligned}
$$

then the crucial property (6) allows us to derive the following self-consistent equation for $G(z)$ :

$$
G(z)=G_{0}\left(z-R_{1}(G(z))\right)
$$

where $R_{1}(z)$ is a kind of self-consistent self-energy for $H_{1}$, namely it is defined by

$$
G_{1}(z)=\frac{1}{z-R_{1}\left(G_{1}(z)\right)}
$$

with $R_{1}(0)=0$. In the special case of a semi-circle eigenvalue distribution for $f, G_{1}$ is given by

$$
G_{1}(z)=\frac{z-\sqrt{z^{2}-4 \sigma^{2}}}{2 \sigma^{2}}=\frac{1}{z-\sigma^{2} G_{1}(z)}
$$

showing that $R_{1}(z)=\sigma^{2} z$. In this case our formula (12) reduces to Wegner's result [4, 15]

$$
G(z)=G_{0}\left(z-\sigma^{2} G(z)\right)
$$

Given $G(z)$ and $R_{1}(z)$, one can derive linear selfconsistent equations for the one-particle and two-particle Green's functions. Again, the freeness property (6) is the essential ingredient for closing the equations. For the Fourier-transform of the one-particle Green's function one obtains

$$
\tilde{G}(q ; z)=\frac{1}{z-v(q)-R_{1}(G(z))}
$$

with $v_{r}=\int_{q} v(q) e^{i q r}$ where $\int_{q}=\frac{\mathcal{V}}{(2 \pi)^{d}} \int_{1 B Z} d^{d} q, \mathcal{V}$ being the volume of the first Brillouin zone $(1 B Z)$. For $R_{1}(z)=$ $\sigma^{2} z$ this reduces again to Wegner's result [4, 15 .

The two-particle Green's function determines the conductivity as a velocity-velocity correlation function. Although in general the two-particle Green's function contains an additional term, one obtains by the symmetry argument $v_{-r}=v_{r}$ that only the product of the oneparticle Green's functions contribute. Thus one finds for zero temperature and in the dc-limit $\omega \rightarrow 0$

$$
\sigma(\omega, 0)=\frac{2 \pi e^{2} B}{\mathcal{V}} \varrho^{2}\left(R_{1}(G) ; E_{F}\right)
$$

where

$$
B:=\int_{q} \frac{|\nabla v(q)|^{2}}{\left|v(q)-\zeta\left(E_{F}+\omega+i 0^{+}\right)\right|^{2}\left|v(q)-\zeta\left(E_{F}+i 0^{+}\right)\right|^{2}}
$$

with $\zeta(z)=z-R_{1}(G(z))$ and the spectral function

$$
\varrho\left(R_{1}(G) ; E\right)=-\frac{1}{\pi} \operatorname{Im} R_{1}\left(G\left(z=E+i 0^{+}\right)\right) .
$$

For $R_{1}(z)=\sigma^{2} z$ this reduces to Wegner's result in a form given by Khorunzhy and Pastur [15]. 
The generality of our solution may be seen from the fact that it includes also the Lloyd model as a special case. Namely, choose a Cauchy-distribution with parameter $\gamma$ as eigenvalue distribution for $f$. Then

$$
G_{1}(z)=\frac{1}{\pi} \int_{-\infty}^{+\infty} \frac{\gamma}{\gamma^{2}+t^{2}} \frac{1}{z-t} d t=\frac{1}{z-i \gamma}
$$

hence $R_{1}(z)=i \gamma$. This shows that the Cauchydistribution behaves in all relevant aspects like an imaginary $\delta$-distribution. For $\delta$-distributions, however, there is no difference between our model and the original Anderson Hamiltonian (see also [16,17]). Since the Anderson model with Cauchy-distributed disorder is nothing else but the Lloyd model, the latter is included in our investigations. In particular, we recover from (12) the $1 \mathrm{PG}$ of the Lloyd model [2]

$$
G(z)=G_{0}(z-i \gamma)
$$

One surprising feature of the Wegner model is that its solution coincides with a special CPA-solution. This generalizes also to our model. In general, the CPA-solution for the Anderson model with single-site random variable $X$ is given by the two equations

$$
G(z)=G_{0}(z-\Sigma(z))
$$

and

$$
\left\langle\frac{X-\Sigma(z)}{1-(X-\Sigma(z)) G(z)}\right\rangle=0 .
$$

The first of these equations coincides with our solution (12) if we identify $\Sigma(z)=R_{1}(G(z))$. By using the equivalent form of (13), namely

$$
G_{1}\left(R_{1}(z)+z^{-1}\right)=z
$$

it can be checked that our solution fulfills also (22) if we choose as distribution for $X$ the eigenvalue distribution of $f$. Note that we specify our model rigorously in the beginning and that we are able to calculate all quantities without any further approximation. Thus our multi-site model is a rigorous mean-field model for the usual singlesite CPA. One should also note that our previous remarks about the Lloyd model can now be taken as an explanation for the well-known fact that CPA is exact for the Lloyd model.

As an instructive example of our formalism let us consider the one-dimensional lattice with nearest-neighbour interaction and binary site-diagonal disorder for the special case $v=\sigma=1$, where $2 v$ is the half-band-width and $\sigma^{2}$ is the variance of the disorder. Then we have $G_{0}(z)=1 / \sqrt{z^{2}-1}$ and $R_{1}(z)=\left(\sqrt{1+z^{2}}-1\right) / 2 z$, which yields as a solution of (12)

$$
G(z)=\frac{8}{6 \sqrt{z^{2}-2}+2 z} .
$$

This provides

$$
\varrho\left(R_{1}(G) ; E\right)=\frac{1}{4 \pi} \sqrt{2-E^{2}} \Theta(\sqrt{2}-E),
$$

i.e. together with (17) a finite conductivity everywhere inside the band $[-\sqrt{2}, \sqrt{2}]$.

Since we can prescribe in our model an arbitrary distribution for $f$ - or for $X$ in the CPA-formulation -, it is clear that our formalism is capable of covering a lot of quite different examples, like, e.g., a superposition of binary noise or, more general, the recently investigated $q$ noise 18]. Furthermore, our description using the theory of free random variables and the notion of non-crossing cumulants allows a straightforward generalization to the case of dynamical disorder and thus promises to give a rigorous model for dynamical CPA. These subjects will be pursued further in forthcoming investigations.

We acknowledge helpful discussions with Franz Wegner and Petr Chvosta. This work was supported by the Deutsche Forschungsgemeinschaft (R.S.).

$\dagger \quad$ Supported by a fellowship from the DFG

[1] P.W. Anderson, Phys. Rev. 109, 1492 (1958)

[2] P. Lloyd, J. Phys. C 2, 1717 (1969)

[3] I.M. Lifshitz, S.A. Gredeskul, and L.A. Pastur, Introduction to the theory of disordered systems (Wiley, New York, 1988)

[4] F. Wegner, Phys. Rev. B 19, 783 (1979)

[5] D. Voiculescu, Invent. math. 104, 201 (1991)

[6] D. Voiculescu, K. Dykema, and A. Nica, Free Random Variables (AMS 1992)

[7] R. Speicher, Math. Ann. 298, 611 (1994)

[8] P. Neu and R. Speicher, Z. Phys. B 92, 399 (1993)

[9] E.P. Wigner, Ann. Math. 62, 548 (1955); 67, 325 (1958)

[10] L. Arnold, J. Math. Anal. Appl. 20, 262 (1967)

[11] R. Speicher, RIMS 29, 731 (1993)

[12] R. Speicher, Prob. Th. Rel. Fields 84, 141 (1990)

[13] D. Voiculescu, Lecture Notes in Mathematics 1132, 556 (1985)

[14] D. Voiculescu, J. Funct. Anal. 66, 323 (1986)

[15] A.M. Khorunzhy and L.A. Pastur, Commun. Math. Phys. 153, 605 (1993)

[16] H. Maassen, J. Funct. Anal. 106, 409 (1992)

[17] H. Bercovici and D. Voiculescu, Indiana U. Math. J. 42, 733 (1993)

[18] P. Neu and R. Speicher, Z. Phys. B 95, 101 (1994) 